\title{
Condiciones objetivas y subjetivas de trabajo y trastornos psicofísicos
}

\author{
Objective and subjective working conditions \\ and psychophysical disorders
}

Margarita Pulido-Navarro 1

Mariano Noriega-Elío 2

\footnotetext{
1 Hospital General de Zona 24, Instituto Mexicano del Seguro Social. Insurgentes Norte 1322, Col. Magdalena de las Salinas, Delegación Gustavo A. Madero, 07760, México, D. F. marpulido7@hotmail.com 2 Maestría en Ciencias de la Salud en el Trabajo, Universidad Autónoma Metropolitana-Xochimilco. Calzada del Hueso 1100, Col. Villa Quietud, Delegación Coyoacán, 04960, México, D. F. mnoriega@cueyatl.uam.mx
}

\begin{abstract}
The relationship between working conditions, psychophysical disorders, and employees' attitudes towards their jobs were analyzed in a pharmaceutical factory in Mexico. A crosssectional study was conducted using three instruments: guided observation of job processes, guided evaluation of health and safety conditions, and an epidemiological questionnaire ( $N=$ 377). Tasks were found to be automated to a considerable degree, but organization was basically Fordist. Strong associations were found between sets of job requirements and certain groups of health outcomes. In the study population, perception of the job (attitude, satisfaction, control, and social support) showed an association with certain health problems. The study concluded that both subjective and objective occupational conditions play an important role in workers' health.
\end{abstract}

Key words Work; Occupational Health; Psychophysical Disorders

Resumen Se analiza la relación entre las condiciones laborales, los trastornos psicofísicos y la valoración sobre el contenido del trabajo en una industria químico farmacéutica en México. Se llevó a cabo un estudio transversal a través de la aplicación de tres instrumentos: una guía de observación del proceso de trabajo, una guía para valorar las condiciones de seguridad e higiene en la empresa y una encuesta epidemiológica $(N=377)$. Los resultados muestran un proceso laboral muy automatizado, pero con una organización del trabajo principalmente fordista. Se encontraron fuertes asociaciones entre conjuntos de exigencias y conjuntos patológicos. La percepción que la población en estudio tiene de su trabajo (valoración, satisfacción, contenido, apoyo social) muestra relación con algunos daños a la salud. Se pudo concluir que tanto las condiciones objetivas de trabajo como las subjetivas juegan un papel importante en la generación de enfermedades en los trabajadores.

Palabras-clave Trabajo; Salud Ocupacional; Trastornos Psicofísicos 


\section{Introducción}

Con el advenimiento de nuevas formas de organización y cada vez más sofisticadas tecnologías introducidas en los centros de trabajo, el entorno de los trabajadores y los procesos laborales se han visto paulatinamente modificados. La actividad industrial se ha transformado a nivel mundial a raíz de la crisis de las dos últimas décadas y por el desmedido y desigual proceso globalizador. La respuesta de las potencias consiste en una serie de cambios, tanto económicos como sociales, que buscan crear las condiciones para lograr un nuevo modelo de acumulación de capital y establecer el llamado nuevo orden económico internacional. En particular, en México, este proceso de "modernización" ha modificado sustancialmente la política económica y social. A partir de él se aplican las estrategias neoliberales del nuevo modelo basadas en la "flexibilidad productiva" (Tamez, 1993).

La integración económica mundial a través de las empresas transnacionales no ha podido desarrollar un progreso social equiparable al lucro que crea. No ha eliminado las contradicciones inherentes al capital, sólo modifica sus formas y oscurece así las relaciones sociales. El crecimiento acelerado de ganancias, propiciado por la innovación tecnológica, ante un mercado limitado, ha impulsado a las empresas de las grandes potencias a trascender sus límites geográficos.

Este proceso en la industria farmacéutica en México asume características muy particulares. La modernización y desarrollo de las empresas transnacionales, con un fuerte avance en la investigación y en las técnicas de mercado, dentro de un marco centralizado, han tenido ventajas monopólicas sobre las firmas nacionales mexicanas, a través del desarrollo de nuevos productos y procesos de producción. La rentabilidad de la industria farmacéutica es muy alta, con grandes márgenes de ganancias incrementados aún por los paraísos fiscales, que minimizan las cargas de impuestos a este tipo de empresas. La principal razón, en las últimas décadas, de sus grandes lucros estriba en la sobrefacturación de sus substancias activas, que los países en desarrollo importan de la empresa transnacional (Soria, 1980).

La empresa en estudio, como otras farmacéuticas transnacionales, aprovecha una serie de ventajas para su expansión mundial. En nuestro país utiliza las instalaciones de otras industrias - ya bien constituidas - al absorberlas dentro de su monopolio o, con su gran poder adquisitivo, compra una planta que ha ob- tenido certificaciones de calidad, lo que le ahorra la implementación propia. La constante innovación tecnológica, con la consecuente modernización de sus procesos industriales le garantiza un amplio margen de ganancias con la menor posibilidad de riesgos para la producción.

Tres políticas particulares definen las iniciativas de esta empresa: innovación, rapidez y crecimiento superiores, con un fin primordial: el rendimiento máximo. A partir de estas políticas se estructuran una serie de cambios permanentes que finalmente tienen repercusión sobre el último eslabón de la cadena de decisiones: el trabajador, quien únicamente asume las directrices dictadas desde los niveles jerárquicos más altos. De ahí también que el obrero sea considerado sólo un factor más para asegurar la producción y que sus condiciones laborales no sean las más propicias para su desarrollo.

La tendencia hacia la mecanización y la automatización incrementa la demanda psíquica de los trabajadores, al someterlos a cambios que implican simplificación, monotonía y repetitividad de la tarea y, sobre todo, a actividades carentes de contenido e interés. Las consecuencias de estos cambios se manifiestan en trastornos mentales, asociados con el estrés y la fatiga y, con frecuencia, en la aparición de una gran variedad de enfermedades físicas (Gardell, 1982; Glaser et al., 1987; Laurell, 1983; Noriega, 1993; Schnall et al., 1992, 1998). Además, se ha hecho evidente el incremento del estrés psicológico en las poblaciones trabajadoras (Bourbonnais et al., 1998) y de fatiga patológica que, aunque algunos la asocian a factores infecciosos y físicos (Chen, 1986; Yousef et al., 1988), muchos otros la asocian a aspectos sociales y laborales (Pawlikowska et al., 1994).

Esta enajenación del trabajo, aunque más refinada que en el taylorismo y el fordismo de las primeras décadas del siglo pasado, produce también una percepción negativa en los trabajadores que genera sufrimiento mental y físico, derivado de la modernización económica (Kawakami, 1993).

\section{Metodología}

Se llevó a cabo un estudio de tipo transversal con la aplicación de tres instrumentos: (a) una guía de observación del proceso de trabajo, que incluyó la indagación sobre las diferentes etapas del proceso productivo, sus áreas, departamentos y puestos de trabajo, así como sus principales formas de organización laboral (turnos, rotación de turnos, trabajo nocturno, extensión de la jornada y pausas) y la construc- 
ción de un diagrama de flujo del proceso; (b) una guía para evaluar las condiciones de seguridad e higiene en la empresa, que examinó 121 preguntas divididas en 16 capítulos: edificios, locales e instalaciones de la empresa; orden y limpieza; sistemas contra incendios; instalaciones eléctricas; manejo, transporte y almacenamiento de materiales; señales, avisos de seguridad y código de colores; ruido; vibraciones; radiaciones ionizantes y no ionizantes; condiciones térmicas extremas; ventilación; iluminación; herramientas, equipos y maquinaria; agentes contaminantes biológicos; materiales y sustancias químicas peligrosas y servicios para los trabajadores (Franco, 1998) y (c) una encuesta individual para ser cumplimentada personalmente, la cual se aplicó a 377 trabajadores de un universo de 412 , lo que significa una no respuesta del $8.5 \%$. Este instrumento recogió algunos aspectos demográficos y de condiciones de vida, así como los riesgos y exigencias más importantes detectados por los otros dos instrumentos. Además, captó la presencia de indicadores relacionados con el contenido y el control del trabajo y sobre la valoración de los trabajadores con respecto al mismo, tales como: posibilidad de fijar el ritmo de trabajo, aprendizaje de nuevos conocimientos, desarrollo de habilidades, creatividad, toma de decisión, satisfacción laboral y apoyo social. Sobre los daños a la salud se indagaron aspectos relacionados con las condiciones de trabajo, con especial interés en los trastornos somáticos (hipoacusia, musculoesqueléticos, varices); mentales (ansiedad, depresión, trastornos del sueño), psicosomáticos (digestivos, cardiovasculares, cefalea, migraña) y fatiga. Los trastornos irritativos, tanto dermatológicos, de vías respiratorias como de conjuntivas, no se abordaron debido a su menor frecuencia, probablemente asociada a una aparición tardía de estos padecimientos por su largo período de exposición y de latencia, que no se presentaron de manera tan importante como los otros padecimientos, por la alta movilidad de estos trabajadores.

La investigación se realizó durante los años 2000 y 2001. El trabajo de campo se hizo en el año 2000, en los meses de julio (aplicación de las guías) agosto y septiembre (aplicación de la encuesta). El acceso a la empresa y la relación con los trabajadores se hizo a través del servicio médico. Éste es contratado por la propia empresa y tiene más de cinco años prestando un adecuado servicio, lo que en la actualidad hace que los trabajadores tengan relativa confianza en él. Además, se advirtió a los trabajadores que los datos, en ningún caso, se usarían de manera personal o se tendría acceso por parte de la empresa a los mismos, sino que su uso sería sólo para fines epidemiológicos y estadísticos. Por estas dos razones se puede suponer que no hay un sesgo importante en las respuestas derivadas de la encuesta.

\section{Resultados}

La población en estudio tiene en promedio 33 años, con una desviación estándar de 7.2. Dos terceras partes son del sexo masculino. Casi la tercera parte tiene carrera universitaria y el $75 \%$ completó, al menos, la educación secundaria. El $64 \%$ es casado y el $69 \%$ tiene hijos. Estas características nos hablan de una población preparada, con relativa estabilidad y con necesidad de conservar el empleo como forma de asegurar la subsistencia de la familia.

A través de la aplicación de la guía para evaluar las condiciones de seguridad e higiene se pudo constatar que la empresa cumple, en términos generales, con las directrices fundamentales vigentes, de acuerdo con las normas oficiales mexicanas; esto quiere decir que los llamados riesgos físicos y químicos no rebasan los límites permisibles. Sin embargo, las normas oficiales no contemplan todos los aspectos relacionados con la propia actividad de los trabajadores y con las formas de organización del trabajo que impone la empresa. Ambos aspectos, como se muestra en los resultados, presentan complicaciones y tienen fuertes repercusiones en la salud de los trabajadores. Incluso aquellos elementos que están dentro de la normatividad afectan a los operarios, como es el caso del ruido que, como se sabe, con límites inferiores a los permitidos también causa problemas diversos mentales y físicos.

El campo de actividad de la empresa incluye dos líneas principales: fabricación de medicamentos y vitaminas. Cuenta con dos plantas: una de ellas se dedicada a la fabricación de antibióticos y su producción es mecanizada. La otra, fabrica diversos productos farmacéuticos como analgésicos, esteroides, antihipertensivos y antitusígenos, entre otros. Los procesos en esta planta son, en su mayor parte, automatizados.

Los resultados recogidos en la guía de observación muestran que el proceso productivo en la empresa incorpora una tecnología muy avanzada, donde se observa una organización fordista de la producción, a pesar de que el proceso está muy automatizado. El ritmo que se imprime a las actividades adquiere diferentes matices dependiendo de las necesidades de la producción. Así, hay periodos en los que se 
trabaja a ritmos muy acelerados para alcanzar las metas de producción fijadas por la empresa. Durante el periodo de observación, el ritmo no era acelerado, lo que daba la impresión de lentitud y aburrimiento en los operarios. La modalidad fordista y la automatización dan pie a la repetitividad y parcelación de las tareas, con una simplificación extrema del trabajo. Su función se limita en el mejor de los casos a alimentar la máquina una y otra vez con materias primas, frascos, cápsulas, grageas, cajas individuales, pero en otros, su actividad queda restringida a la vigilancia pasiva de la máquina. El obrero no tiene la menor posibilidad de participar en la planeamiento y organización de las tareas. Así, se evidencia un trabajo carente de contenido que, lejos de favorecer el desempeño de las capacidades de los trabajadores, obstaculiza el desarrollo de habilidades y potencialidades y la adquisición de nuevos conocimientos. Tampoco les brindan la posibilidad de plantearse metas a alcanzar en su propio trabajo, más bien los despojan de su capacidad de raciocinio, pues no necesitan resolver situaciones que requieran análisis, juicio, cálculo, abstracción; en resumen, el uso de las funciones mentales superiores (Guélaud et al., 1981). Hay un riguroso control de calidad de los productos donde los trabajadores verifican que éstos cumplan con las características de calidad requeridas. En esta fase del proceso los trabajadores están sujetos a una supervisión muy estricta, alta responsabilidad y alto ritmo de trabajo.

Un aspecto trascendente, en cuanto a la forma de organización del trabajo, es la rotación de turnos. La rotación afecta la salud de los tra- bajadores al verse alterado su ciclo circadiano y su vida cotidiana en el ámbito individual, familiar y social. Además, la empresa impone mayor tensión al añadir un ingrediente de inseguridad porque los supervisores, ya sea por necesidades de la producción, por favoritismo o como mecanismo de represión, pueden decidir cuándo y cómo modificar el turno de cualquier trabajador, problema que además genera división y rivalidad entre los obreros, asegurando para los supervisores un gran poder.

La empresa muestra poco interés en las condiciones de sus trabajadores, ya que existe poca permanencia de los mismos en ella. Existe alta movilidad de los operarios, puesto que, a pesar de que la empresa tiene 20 años en la zona, el $46 \%$ de los trabajadores no alcanza 6 años de antigüedad. A pesar de eso, por las condiciones de exigencia hay poco ausentismo. En el último año, sólo la tercera parte de la población faltó en alguna ocasión y alrededor del $30 \%$ de ellos faltaron por motivos de salud. En promedio, las ausencias por ésta y otras causas fueron de entre uno y dos días.

Para mostrar los hallazgos más relevantes del perfil epidemiológico general se presentan las principales asociaciones entre los riesgos, las exigencias y los problemas de salud. Como se observa en la Tabla 1 es evidente que en estos trabajadores predomina la exposición a las exigencias sobre los riesgos y, además, que hay una mayor asociación de éstas con los trastornos y las enfermedades. Sin embargo, es importante aclarar que los riesgos físicos y químicos, además de seguir teniendo importancia en esta industria, pueden no mostrar una asociación tan evidente con las enfermedades, como

Perfil epidemiológico del conjunto de trabajadores de la empresa.

\begin{tabular}{|c|c|c|}
\hline Perfil de riesgos y exigencias & Tasa* & Asociación ${ }^{\star \star}$ \\
\hline Control estricto & 70,6 & Psicosomáticos digestivos y musculoesqueléticos \\
\hline Minuciosidad & 56,0 & Ansiedad, musculoesqueléticos y fatiga \\
\hline $\begin{array}{l}\text { Inmovilidad (posición incómoda, } \\
\text { permanecer de pie) }\end{array}$ & 42,4 & $\begin{array}{l}\text { Trastornos musculoesqueléticos, ansiedad, trastornos } \\
\text { del sueño, fatiga y varices }\end{array}$ \\
\hline Jornada prolongada & 41,1 & Ansiedad \\
\hline Ruido & 39,3 & $\begin{array}{l}\text { Depresión, ansiedad, trastornos del sueño, migraña, fatiga } \\
\text { e hipoacusia }\end{array}$ \\
\hline Repetitividad & 38,7 & Migraña, fatiga y trastornos musculoesqueléticos \\
\hline $\begin{array}{l}\text { Trabajo en días de descanso } \\
\text { y vacaciones }\end{array}$ & 35,5 & Gastritis, úlcera, colitis \\
\hline
\end{tabular}

* Tasa por 100 trabajadores.

** Mantel-Haenszel $\mathrm{p}<0.05$

Fuente: Encuesta individual, México, 2000. 
es el caso de las exigencias, debido a que la aparición de estos padecimientos ocurre después de largo tiempo de exposición y de latencia, lo que difícilmente se puede ver en esta población que tiene alta movilidad.

Las exigencias son básicamente de tres clases: las relacionadas con el tipo de tareas, cuya característica principal es la falta de contenido del trabajo (minuciosidad, inmovilidad, repetitividad); las asociadas al tiempo de trabajo (jornada prolongada y trabajo en días de descanso y vacaciones) y, por último, las relacionadas con una supervisión y control muy estrictos. En relación con los riesgos el más importante es el ruido. Como se observa, estas exigencias desempeñan un papel trascendente en la aparición de dos conjuntos patológicos: los psíquicos, los psicosomáticos y la fatiga (tasa de 69 por cada 100 trabajadores) y los musculoesqueléticos (tasa de 48 por 100). Se puede decir que éstos dos conjuntos son los que definen el perfil de estos trabajadores (Tabla 1).

\section{Relación entre conjuntos de exigencias} y conjuntos patológicos

Una forma de expresar la complejidad de los procesos de trabajo es a través de analizar su impacto en conjuntos de exigencias. El estudio mostró asociaciones significativas entre manifestaciones como la fatiga y los trastornos musculoesqueléticos con estos conjuntos. Lo que se pretende mostrar es que - a pesar de la visión de la medicina del trabajo, centrada todavía hoy en día en un enfoque unicausal -, los problemas de salud se explican por conjuntos de elementos que comparten un origen común en cuanto a sus determinantes.

Así, en la Tabla 2, el conjunto de exigencias asociadas a la fatiga patológica, tales como, el trabajo monótono o aburrido, el exceso de atención, la inmovilidad, las posiciones incómodas y el permanecer de pie durante toda la jornada, evidencian un tipo de actividad carente de control y de contenido y con múltiples problemas de tipo ergonómico. Todos los trabajadores expuestos presentan un riesgo del doble al cuádruple de presentar fatiga que aquéllos no expuestos.

Del mismo modo, la Tabla 3 muestra que los trastornos musculoesqueléticos también están estrechamente asociados con exigencias ergonómicas y con una actividad carente de contenido. Los trabajadores que padecen este tipo de situación tienen aproximadamente el doble de riesgo de presentar estos problemas de salud.

Integralmente, puede decirse que en el proceso de trabajo de la industria farmacéutica, con alto desarrollo tecnológico, predomina un tipo de trabajador descalificado, con tareas muy parceladas, desprovistas de control y contenido, que se caracterizan por exigencias que impiden un desarrollo ergonómico adecuado y que se expresan en problemas como la fatiga y los trastornos musculoesqueléticos.

\section{Condiciones subjetivas de trabajo y salud}

Dentro del campo de la subjetividad en el trabajo se analizaron cuatro elementos fundamentales: la valoración que el trabajador hace su actividad y las condiciones en las cuales se desarrolla ésta; la forma en que percibe si hay satisfacción o no en función de lo que hace en su actividad productiva; cómo valora sus tareas en relación con si éstas tienen control o carecen de él; y si cuenta con apoyo social dentro del trabajo para afrontar las situaciones que se le presentan. Estas condiciones se analizaron

Relación entre fatiga y exigencias laborales.

\begin{tabular}{lccc}
\hline Exigencia & $\begin{array}{c}\text { Razón de } \\
\text { prevalencia* }\end{array}$ & $\begin{array}{c}\text { Intervalo de } \\
\text { confianza 95\% }\end{array}$ & $\mathbf{p}^{\star *}$ \\
\hline Trabajo aburrido & 3,8 & $1,4-10,4$ & 0,04 \\
No poder desatender la tarea & 3,4 & $1,7-7,0$ & 0,003 \\
Posiciones incómodas & 2,6 & $1,4-5,0$ & 0,003 \\
Permanecer de pie & 2,5 & $1,3-5,0$ & 0,005 \\
Estar fijo en el lugar de trabajo & 2,3 & $1,2-4,5$ & 0,01 \\
\hline
\end{tabular}

* Tasa de expuestos entre tasa de no expuestos.

** Mantel-Haenszel y, en trabajo aburrido, Prueba Exacta de Fisher.

Fuente: Encuesta individual, México, 2000. 
por medio de la construcción de cuatro índices, uno para cada condición mencionada.

En la percepción de los trabajadores no sólo se trató de captar la valoración sobre el contenido del trabajo, sino que se pretendió introducir la idea sobre si hay propiamente elementos humanizantes en el trabajo, es decir, si éste es estimulante para la creación y recreación del propio sujeto.

La percepción negativa que tienen los trabajadores por las actividades repetitivas y carentes de contenido también se expresa en la aparición de sufrimiento mental y físico. Así, esta percepción muestra relación con algunos daños a la salud. Se observaron riesgos desde dos a cuatro y medio veces más altos de trastornos psíquicos y de fatiga en los trabajadores con percepción negativa respecto a la valoración del trabajo, satisfacción en el trabajo, control sobre el trabajo y apoyo social que en aquellos con percepción positiva (Tabla 4).

Podría parecer que la subjetividad de los trabajadores, entendida como la percepción que tienen de su actividad laboral, al contrario de lo que se piensa comúnmente, influye menos en los trastornos psicosomáticos que en los psíquicos y la fatiga. Así se observa en la Tabla 4 que los psicosomáticos son los que menos diferencias presentaron entre los expuestos y los no expuestos.

Se pudo apreciar que un índice muy sensible es el del apoyo social, ya que mostró notables diferencias entre aquellos trabajadores que lo tienen y los que no, es decir, aquéllos en los que sus compañeros de trabajo son solidarios y valoran lo que hacen y también en los que sus jefes consideran que las tareas que lleva a cabo el trabajador son importantes. El sistema de apoyos sociales en estos trabajadores puede estar amortiguando las respuestas al estrés y protegiéndolos contra los efectos dañinos de éste.

\section{Discusión}

La metodología de investigación utilizada, que combina técnicas cualitativas (guía de observación del proceso de trabajo y guía de seguridad e higiene) y cuantitativas (encuesta), permitió tener una visión integral de la relación entre las condiciones laborales y de salud en esta empresa y en este grupo de trabajadores. La visión cualitativa permite conocer las características de los procesos de trabajo y de las condiciones de seguridad e higiene y, por lo tanto, permite proponer cuáles son los aspectos centrales que deben ser estudiados en cada uno de los trabajadores. Reducirse sólo a una de estas técnicas quita riqueza a la información y por lo tanto a un entendimiento más integral de lo que es el trabajo y la salud en un colectivo.

En este centro de trabajo se encontraron en condiciones aceptables muchos aspectos de seguridad e higiene, tales como: áreas de trabajo; sistemas contra incendio; manejo, transporte y almacenamiento de materiales y sustancias químicas peligrosas; señales, avisos y códigos de colores; herramientas, equipos y maquinaria; así como los servicios para los trabajadores. Es decir, se satisfacen los estándares de seguridad definidos en la norma ISO-9000. Además, también se cuenta con varios elementos favorables exteriores al trabajo, como son: extensas áreas verdes y canchas deportivas. Desafortunadamente, estas medidas se centran más en la búsqueda de la superación de la calidad del producto que en la preocupación por las condiciones de trabajo y de salud de las personas que ahí laboran.

Los resultados obtenidos muestran que los problemas de salud que afectan a los trabajadores son derivados de las formas de organización asumidas en los procesos laborales. La automatización implica poco control de estos procesos por parte de los trabajadores y una medición rigurosa del rendimiento individual con muchas otras consecuencias, tales como, la desaparición de la necesidad de desplazarse para acercar materiales o alimentar a la máquina, lo que reduce los tiempos "improductivos". El ritmo de trabajo se sigue supeditando a la máquina, lo que limita más la autonomía. La automatización reduce las tareas, pero esta reducción de funciones trae cargas psíquicas (Rajchenberg, 1990).

Los trabajadores en este estudio presentaron, a pesar de su condición de sanos, una serie de síntomas que en inicio hablan de sufrimiento, indicios orientadores del malestar reinante, probable generador o disparador de entidades nosológicas bien reconocidas y prevenibles (Gardell, 1982; Manuck et al., 1995; Rosengren, 1991) y que tienen una alta incidencia en los trabajadores, en gran parte debida a las características de los actuales procesos productivos. En la mayor parte de las actividades analizadas, el trabajador no puede ejercer control sobre sus tareas, ni tiene la posibilidad de incrementar sus conocimientos o desarrollar su creatividad e iniciativa. Así, se mantiene latente la evocación de afectos negativos, listos a dispararse justo en el momento de agregar tensión a la actividad (Karasek \& Theorell, 1990).

Muchos de los trabajadores encuestados mostraron una percepción positiva de su acti- 
Relación entre trastornos musculoesqueléticos y exigencias.

\begin{tabular}{lccc}
\hline Exigencia & $\begin{array}{c}\text { Razón de } \\
\text { prevalencia* }\end{array}$ & $\begin{array}{c}\text { Intervalo de } \\
\text { confianza 95\% }\end{array}$ & $\mathbf{p}^{\star \star}$ \\
\hline Estar fijo en el lugar de trabajo & 2,8 & $1,5-5,2$ & 0,0007 \\
Permanecer de pie & 2,1 & $1,2-3,8$ & 0,01 \\
Posiciones incómodas & 2,0 & $1,1-3,6$ & 0,02 \\
No poder desatender la tarea & 1,9 & $1,1-3,3$ & 0,01 \\
Supervisión estricta & 1,8 & $1,1-3,0$ & 0,03 \\
\hline
\end{tabular}

* Tasa de expuestos entre tasa de no expuestos.

** Mantel-Haenszel.

Fuente: Encuesta individual, México, 2000.

Tabla 4

Relación entre condiciones subjetivas de trabajo y daños a la salud *.

\begin{tabular}{lcccc}
\hline Daños & Valoración & Satisfacción & Control & Apoyo social \\
\hline Psíquicos & 3,1 & 1,9 & 3,8 & 4,5 \\
Psicosomáticos & 1,5 & 1,1 & 1,2 & 2,9 \\
Fatiga & 3,6 & 1,8 & 2,6 & 2,9 \\
\hline
\end{tabular}

* Tasa de los que contestaron negativamente entre tasa de los que contestaron positivamente.

Fuente: Encuesta individual, México, 2000.

vidad, a pesar de que su labor no les depara la posibilidad de desarrollo, situación que nos puede hablar de cierto grado de enajenación de su condición humana. Esta condición busca siempre la forma de plasmar sus potencialidades; el trabajo representa el principal recurso del hombre para lograr ese objetivo, pero si ese recurso está bloqueado, presenciamos entonces una subutilización de las capacidades mentales diseñadas para tal fin.

Ante la idea generalizada de que los trabajadores prefieren un trabajo simple, que no les represente esfuerzo mental, hay estudios que demuestran lo contrario, es decir, que los operarios prefieren actividades enriquecidas (Guélaud et al., 1981). En esta idea juega un peso muy importante el juicio histórico respecto al trabajo, es decir, la consideración del trabajo como algo desagradable y forzoso. La subutilización de las estructuras mentales ocasiona, tarde o temprano, la esclerosis de las estructuras cerebrales destinadas al pensamiento abstracto. Hasta el momento no se ha llevado a cabo intento alguno por indemnizar a los trabajadores por esa disminución en sus capacidades funcionales. De otra manera, los que se en- cargan de organizar las tareas tendrían más cuidado en dotar de contenidos enriquecedores todas las actividades laborales.

Una percepción negativa del trabajo se traduce en insatisfacción y frustración de las potencialidades de los trabajadores que da lugar a disgusto, tensión y manifestaciones psíquicas y somáticas de daño (Frankenhaeuser, 1991). Sin embargo, los trabajadores que perciben esa situación los coloca en posición de oponer resistencia. Podría incluso ese malestar, jugar un papel de mecanismo de defensa de su integridad, cuyo rasgo principal es el raciocinio. Así, en situaciones que ponen en riesgo esa cualidad esencial, la llamada de atención sería el síntoma psíquico o físico para evitar o superar la situación.

A muchos de los trabajadores estudiados, las condiciones laborales no les ofrecen la oportunidad de desarrollar sus capacidades. Las ideas de un trabajo "saludable" enfatizan la necesidad de dar posibilidades a los trabajadores de: influir y controlar sus actividades, experimentar sentimiento de comunidad y pertenencia en el trabajo y desarrollar capacidades personales y aptitudes profesionales por me- 
dio de aprendizaje continuo (Frankenhaeuser \& Johansson, 1986). La valoración de las exigencias por parte del trabajador lo obliga a estimarlas en relación con sus capacidades de afrontamiento (Frankenhaeuser, 1989), por eso el control personal y un margen en la toma de decisiones se consideran factores de amortiguación que permiten a las personas trabajar mucho y a la vez disfrutar del trabajo y conservar su salud (Karasek \& Theorell, 1990). El ejercer control en el trabajo, entendido éste como la capacidad de los trabajadores para influir en los sucesos relacionados con su trabajo, au- menta la satisfacción laboral y reduce las respuestas corporales al estrés. Esa posibilidad de asumir un papel activo y participativo en el trabajo, al permitir utilizar de la mejor manera las capacidades del trabajador, incrementa la autoestima (Frankenhaeuser, 1989). Si no se cuenta con estas condiciones favorables, o aun contando con ellas, una estrategia importante para afrontar el estrés en el trabajo es el apoyo social (Gardell, 1982; Shankar \& Famuyiwa, 1991). De ahí que estos elementos sean esenciales a valorar cuando se estudia la relación entre el trabajo y la salud.

\section{Referencias}

BOURBONNAIS, R.; COMEAU, M.; VÉZINA, M. \& DIONE, G., 1998. Job strain, psychological distress and burnout in nurses. American Journal of Industrial Medicine, 34:20-28.

CHEN, M., 1986. The epidemiology of self perceived fatigue among adults. Preventive Medicine, 15:7481.

FRANCO, J. G., 1998. Verificación, Diagnóstico y Vigilancia de la Salud Laboral en la Empresa. México, DF: Universidad Autónoma Metropolitana-Xochimilco.

FRANKENHAEUSER, M., 1989. A biopsychosocial approach to work life issues. International Journal of Health Services, 19:747-758.

FRANKENHAEUSER, M., 1991. The psychophysiology of workload, stress and health: Comparison between the sexes. Annals of Behavioral Medicine, 13:197-204. 
FRANKENHAEUSER, M. \& JOHANSSON, G., 1986. Stress at work: Psychobiological and psychosocial aspects. International Review of Applied Psychology, 35:287-299.

GARDELL, B., 1982. Scandinavian research on stress in working life. International Journal of Health Services, 12:31-41.

GLASER, R.; RICE, J.; SHERIDAN, J.; FERTE, R.; STOUT, J.; SPEICHER, C. E.; PINSKY, D.; KOTUR, J.; POST, A.; BECK, M. \& KIECOL-GRASER, J. K., 1987. Stress-related immune suppression: Health implications. Brain, Behavior, and Immunity, 1:7-20.

GUÉLAUD, F.; BEAUCHESNE, M.; GAUTRAT, J. \& ROUSTANG, G., 1981. Para un Análisis de las Condiciones de Trabajo en la Empresa. México, DF: Instituto Nacional de Estudios del Trabajo/ Lima: Instituto de Investigación y Desarrollo de la Autogestión.

KARASEK, R. \& THEORELL, T., 1990. Healthy Work Stress. Productivity and the Reconstruction of Working Life. New York: Basic Books.

KAWAKAMI, N., 1993. Effects of job stress on occurrence of mayor depression in Japanese industry a case control study nested in a cohort study. Journal of Educational Psychology, 63:261-270.

LAURELL, C. \& MARQUEZ, M., 1983. Procesos laborales y patrones de desgaste. In: El Desgaste Obrero en México, pp. 14-37, México, DF: ERA.

MANUCK, S.; MARSLAND, A.; KAPLAN, J. R. \& WILLIAMS, J., 1995. The pathogenicity of behavior and its neuroendocrine mediation: An example from coronary artery disease. Psychosomatic Medicine, 57:275-283.

NORIEGA, M., 1993. Organización laboral, exigencias y enfermedad. In: Para la Investigación Sobre la Salud de los Trabajadores, Serie PALTEX 3, pp. 167-187, Washington, DC: Organización Panamaericana de la Salud.
PAWLIKOWSKA, T.; CHALDER, T.; HIRSCH, S.; WALACE, P.; WRIGHT, D. \& WESELY, S., 1994. Population based study of fatigue and psychological distress. BMJ, 308:763-766.

RAJCHENBERG, E., 1990. Nuevas tecnologías, proceso de trabajo y salud. In: Salud y Crisis en México. Textos para un Debate (I. Almada, org.), pp. 157185, México, DF: Siglo XXI.

ROSENGREN, A., 1991. Risk of coronary heart disease in middle-aged male bus and train drivers compared to men in others occupations: A prospective study. International Journal of Epidemiology, 20:82-86.

SCHNALL, P.; SCHWARTZ, J.; LANDSBERGIS, P.; WARREN, K. \& PICKERING, T., 1992. Relation between job strain, alcohol, and ambulatory blood pressure. Hypertension, 19:488-494.

SCHNALL, P.; SCHWARTZ, J.; LANDSBERGIS, P.; WARREN, K. \& PICKERING, T., 1998. A longitudinal study of job strain and ambulatory blood pressure: Results from a three-year follow-up. Psychosomatic Medicine, 60:697-706.

SHANKAR, J. \& FAMUYIWA, O., 1991. Stress among factory workers in a developing country. Journal of Psychosomatic Research, 35:163-171.

SORIA, V., 1980. Estructura y comportamiento de la industria farmacéutica en México. Revista de Ciencias Sociales y Humanidades. UAM Iztapalapa, 1:111-141.

TAMEZ, S., 1993. Flexibilidad Productiva y Accidentes de Trabajo. México, DF: Universidad Autónoma Metropolitana.

YOUSEF, G.; BELL, E.; MANN, G.; MURUGESAN, V.; SMITH, D.; McCARTNEY, R. \& MOWBRAY, J., 1988. Chronic enterovirus infection in patients with postviral fatigue syndrome. Lancet, 1:146150.

Recibido el 27 de noviembre de 2001

Versión final presentada el 21 de junio de 2002

Aprobado el 6 de septiembre de 2002 